\title{
Random Attractors for the Dissipative Hamiltonian Amplitude Equation Governing Modulated Wave Instabilities with Additive Noise ${ }^{*}$
}

\author{
Jinyan Yin, Yangrong Li, Huijun Zhao \\ School of Mathematics and Statistics, Southwest University, Chongqing, China \\ Email: yjy111@swu.edu.cn, liyr@swu.edu.cn, huijun88@swu.edu.cn
}

Received June 10, 2013; revised July 15, 2013; accepted September 10, 2013

Copyright (C) 2013 Jinyan Yin et al. This is an open access article distributed under the Creative Commons Attribution License, which permits unrestricted use, distribution, and reproduction in any medium, provided the original work is properly cited.

\begin{abstract}
In this paper, we study the random dynamical system (RDS) generated by the dissipative Hamiltonian amplitude equation with additive noise defined on the periodic boundaries. We investigate the existence of a compact random attractor for the RDS associated with the equation through introducing two functions and one process in $E_{0}=H^{1} \times L^{2}$. The compactness of the RDS is established by the decomposition of solution semigroup.
\end{abstract}

Keywords: Random Dynamical System; Random Attractor; Hamiltonian Amplitude Equation

\section{Introduction}

The Hamiltonian amplitude equation

$$
\mathrm{i} \phi_{x}+\phi_{t t}+2 \sigma|\phi|^{2} \phi=0
$$

was first proposed by Tanaka, Yajima and Wadati as a model for the nonlinear modulation of stable plane wave in unstable media [1,2]. In 1992 as an improved version of (1), the equation

$$
\mathrm{i} \psi_{x}+\psi_{t t}+2 \sigma|\psi|^{2} \psi-\varepsilon \psi_{x t}=0,0<\varepsilon<1,
$$

was proposed [3], which generalized (1) in the sense that

$$
\psi(x, t ; \varepsilon=0)=\phi(x, t),
$$

but one can show that for most initial data

$$
\lim _{\varepsilon \rightarrow 0} \psi(x, t ; \varepsilon) \neq \phi(x, t),
$$

even if the two functions agree at $t=0$. Both of these models can be derived systematically from more complicated Hamiltonian systems through a particular limiting process (nearly monochromatic waves of small amplitude) corresponding to $\varepsilon \rightarrow 0$. Even so, keeping $\varepsilon \neq 0$ in (2) is crucial because (1) is formally integrable but ill-posed, whereas (2) is a generalization of it which is apparently not integrable but well-posed.

\footnotetext{
*This work is supported by National Natural Science Foundation of China (11071199) and Natural Science Foundation of Chongqing (2009BB8105).
}

In this paper, we consider the following dissipative Hamiltonian amplitude equation governing modulated wave instabilities perturbed by an additive white noise

$$
\begin{gathered}
d u_{t}+\alpha u_{t} d t-\beta u_{x t} d t-\gamma u_{x x} d t+\mathrm{i} u_{x} d t+f\left(|u|^{2}\right) u d t \\
=\sum_{j=1}^{m} h_{j} d W_{j} \\
\quad u(x, \tau)=u_{0}(x), \quad u_{t}(x, \tau)=u_{1}(x),
\end{gathered}
$$

and the periodic boundary condition

$$
u(x-L, t)=u(x+L, t),
$$

where $u$ is an unknown complex valued function, $i$ is the unit of imaginary number, the internal $I=(-L, L)$, $\alpha, \beta$ and $\gamma$ are positive constants, which satisfy $\beta<\gamma$, the functions $h_{j} \in H^{2}(I), j=1,2, \cdots, m$, are time independent, the random functions $W_{j}$, $j=1,2, \cdots, m$, are independent two-side real-valued Wiener processes on a probability space $(\Omega, F, P)$ which will be specified later, and $f(s)$ is $C^{1}$, sf $(s)$ is $C^{2}$ real valued function which satisfies that

$$
\begin{aligned}
& \liminf _{s \rightarrow+\infty} \frac{F(s)}{s^{1+\delta}} \geq \gamma_{0}>0, \quad s \geq \tau, \delta \geq 1, \\
& \lim _{s \rightarrow+\infty} \inf \frac{s f(s)-\vartheta F(s)}{s^{1+\delta}} \geq \gamma_{0}>0, \quad s \geq \tau, \delta \geq 1,
\end{aligned}
$$


where $0<\vartheta<1, \quad \gamma_{0}$ is a constant depended on $\delta$ and $\vartheta$, and $F(s)=\int_{\tau}^{s} f(t) \mathrm{d} t$.

The deterministic case has been studied extensively, for instance, Guo, B. L. and Dai, Z. D. [4] proved that there exists a global weak attractor $\mathrm{A}_{1}$ in $E_{1}=H^{2} \times H^{1}$ for (5) and it is actually a global strong attractor in $E_{1}$. Dai, Z. D. [5] proved the existence of a global attractor $\mathrm{A}_{0}$ in $E_{0}=H^{1} \times L^{2}$, and obtained the equality $\mathrm{A}_{0}=\mathrm{A}_{1}$. Dai, Z. D. Yang, L. Huang, J. [6] obtained a global attractor for the unperturbed system in $E_{0}$ and $E_{1}$ respectively. Yang, L., Dai, Z. D. [7] obtained the estimate of the Hausdorff dimension and the fractal dimension of a global attractor for the perturbed and unperturbed systems separately. However, up to the best of our knowledge, the research for the dissipative Hamiltonian amplitude equation governing modulated wave instabilities with random attractors has not involved.

Recently, many authors have studied the existence of random attractors for other equations [8-10]. In this paper, for (5), we first obtain an absorbing set in $E_{0}$ and $E_{1}$ respectively through introducing two functions and one process, then by the decomposition of solution semigroup we derive the compactness in $E_{0}$. As far as we know, no one has studied stochastic equations through introducing two functions, so this method enriches the study of stochastic equations.

This paper is organized as follows. In Section 2, for convenience of the reader, we recall some basic notions on function spaces and the theory of random dynamical system. In Section 3, we solve Equation (5) and get the corresponding RDS $\varphi$. In Section 4, we prove the existence of a random attractor in $E_{0}$ for this RDS.

Throughout this paper, we adopt the following notations. We write $L^{2}=L^{2}(I), H^{1}=H^{1}(I)$,

$H^{2}=H^{2}(I)$ for short. We denote by $\|$.$\| and || the norms,$ by $((\cdot, \cdot))$ and $(\cdot, \cdot)$ the inner products in $H^{1}$ and $L^{2}$ respectively. We also use $|u|$ to denote the modular or absolute value of $u$.

\section{Preliminaries}

In this section, we recall some basic notions on function spaces [4,7], the theory of RDS [11-14] and introduce the method of the existence of random attractors for the continuous RDS $[8,10]$, which we will use in this paper.

\subsection{Function Spaces and Operators}

We first consider the mathematical setting for (5). Let $L^{2}, H^{1}, H^{2}$ be usual Sobolev space, $E_{0}=H^{1} \times L^{2}$, $E_{1}=H^{2} \times H^{1}$ and $\phi=(u, v)^{\mathrm{T}}$. We define the following scalar products and norms separately:

for any $\phi_{i}=\left(u_{i}, v_{i}\right)^{\mathrm{T}} \in E_{0}$ and $\phi=(u, v)^{\mathrm{T}} \in E_{0}$, we have

$$
\left(\phi_{1}, \phi_{2}\right)_{E_{0}}=\left(\left(u_{1}, u_{2}\right)\right)+\left(v_{1}, v_{2}\right),\|\phi\|_{E_{0}}^{2}=\|u\|^{2}+|v|^{2},
$$

for any $\phi_{i}=\left(u_{i}, v_{i}\right)^{\mathrm{T}} \in E_{1}$ and $\phi=(u, v)^{\mathrm{T}} \in E_{1}$, we have

$$
\left(\phi_{1}, \phi_{2}\right)_{E_{1}}=\left(D^{2} u_{1}, D^{2} u_{2}\right)+\left(\left(v_{1}, v_{2}\right)\right),\|\phi\|_{E_{1}}^{2}=|\Delta u|^{2}+\|v\|^{2}
$$

Let $A=-D^{2}: D(A)=H^{1} \cap H^{2} \rightarrow L^{2}$, then $A$ is a positive self-adjoint operator, which has the first eigenvalue $\lambda_{1}=\inf _{u \in H^{1}} \frac{\|u\|^{2}}{|u|^{2}}$.

\subsection{Random Dynamical Systems}

Let $(\Omega, F, P)$ be a probability space and $\left\{\theta_{t}: \Omega \rightarrow \Omega, t \in R\right\}$ be a family of measure preserving transformations such that $(t, \omega) \rightarrow \theta_{t} \omega$ is measurable, $\theta_{0}=i d$ and $\theta_{t+s}=\theta_{t} \theta_{s}$ for all $s, t \in R$. The flow $\theta_{t}$ together with the corresponding probability space $\left(\Omega, F, \mathrm{P}, \theta_{t}\right)$ is called a measurable dynamical system.

Definition 2.2.1 A continuous random dynamical system(RDS) on a Polish space $(X, d)$ with Borel $\sigma$-algebra on $\left(\Omega, F, \mathrm{P}, \theta_{t}\right)$ is a measurable map

$$
\varphi: R^{+} \times \Omega \times X \mapsto X,(t, \omega, x) \mapsto \varphi(t, \omega) x
$$

such that $\mathrm{P}-$ a.s.

1) $\varphi(0, \omega)=i d$ on $X$;

2) $\varphi(t+s, \omega)=\varphi\left(t, \theta_{s} \omega\right) \varphi(s, \omega)$, for all $s, t \in R^{+}$ (cocycle property);

3) $\varphi(t, \omega): X \mapsto X$ is continuous.

A random compact set $\{K(\omega)\}_{\omega \in \Omega}$ is a family of compact sets indexed by $\omega$ such that for every $x \in \mathrm{X}$ the mapping $x \mapsto d(x, K(\omega))$ is measurable with respect to $F$.

Let $A(\omega)$ be a random set and $B \subset X$. We say $\mathrm{A}(\omega)$ attracts $B$ if

$$
\lim _{t \rightarrow \infty} \operatorname{dist}\left(\varphi\left(t, \theta_{-t} \omega\right) \mathrm{B}, \mathrm{A}(\omega)\right)=0, \mathrm{P}-\text { a.s. } \omega \in \Omega,
$$

where $\operatorname{dist}(\cdot, \cdot)$ denotes the Hausdorff semi-distance in $X$. We say $\mathrm{A}(\omega)$ absorbs $B$ if there exists $t_{B}(\omega)>0$ such that for all $t \geq t_{B}(\omega)$,

$$
\varphi\left(t, \theta_{-t} \omega\right) \mathrm{B} \subset \mathrm{A}(\omega), \mathrm{P}-\text { a.s. } \omega \in \Omega .
$$

A random set $\mathrm{A}(\omega)$ is said to be a random attractor for the $\operatorname{RDS} \varphi$ if $\mathrm{P}-$ a.s.

1) $\mathrm{A}(\omega)$ is a random compact set;

2) $\mathrm{A}(\omega)$ is invariant, that is, $\varphi(t, \omega) \mathrm{A}(\omega)=\mathrm{A}\left(\theta_{t} \omega\right)$ for all $t \geq 0$;

3) $\mathrm{A}(\omega)$ attracts all deterministic bounded sets $B \subset X$.

Theorem 2.2.2 If there exists a random compact set absorbing every bounded set $B \subset \mathrm{X}$, then the RDS $\varphi$ possesses a random attractor $\mathrm{A}(\omega)$,

$$
\mathrm{A}(\omega)=\overline{\mathrm{B} \subset \mathrm{X}} \Lambda_{\mathrm{B}}(\omega),
$$


where $\Lambda_{\mathrm{B}}(\omega):=\cap_{s \geq 0} \overline{\mathrm{\cup}_{t>s} \varphi\left(t, \theta_{-t} \omega\right) \mathrm{B}}$ is the omega-limit set of $B$

\section{Solve the Equation and Generate a RDS}

We consider the probability space $(\Omega, F, P)$, where

$$
\Omega=\left\{\omega=\left(\omega_{1}, \omega_{2}, \cdots, \omega_{m}\right) \in C\left(R, R^{m}\right): \omega(0)=0\right\},
$$

and $F$ is the Borel $\sigma$-algebra induced by the compact open topology of $\Omega$, while $P$ is the corresponding Wiener measure on $(\Omega, F)$. Then, we identify $\omega$ with

$$
W(t)=\left(W_{1}(t), W_{2}(t), \cdots, W_{m}(t)\right)=\omega(t) \text { for } t \in R .
$$

Finally, we define the time shift by $\theta_{t} \omega(\cdot)=\omega(\cdot+t)-\omega(t), \omega \in \Omega, t \in R$. Then $\left(\Omega, F, \mathrm{P},\left(\theta_{t}\right)_{t \in R}\right)$ is a metric dynamical system.

We now want to establish a continuous random dynamical system corresponding to (5). For this purpose, we need to convert the stochastic equation with an additive noise into a deterministic equation with a random parameter.

Given $j=1,2, \cdots, m$, consider the stochastic stationary solution of the one-dimensional Ornstein-Uhlenbeck equation

$$
d z_{j}+\alpha z_{j} d t=d W_{j}(t)
$$
by

One may easily check that a solution to (10) is given

$$
z_{j}(t)=\int_{-\infty}^{t} \mathrm{e}^{-\alpha(t-s)} \mathrm{d} W_{j}(s), t \in R .
$$

Putting $z=\sum_{j=1}^{m} h_{j} z_{j}$, by (10) we have

$$
d z+\alpha z d t=\sum_{j=1}^{m} h_{j} d W_{j}
$$

We also need two facts

$$
\left(E\left|z_{j}(0)\right|\right)^{2} \leq E\left|z_{j}(0)\right|^{2}=\int_{-\infty}^{0} \mathrm{e}^{2 \alpha \tau} \mathrm{d} \tau=\frac{1}{2 \alpha} \rightarrow 0,
$$

as $\alpha \rightarrow \infty$. We also have

$$
\lim _{t \rightarrow \infty} \frac{Z_{j}(t)}{t}=0, \quad \mathrm{P}-\text { a.s. }
$$

Assumed $h_{j} \in D(A) \subset H^{2}$. Then by Sobolev embedding theorem, $H^{2}(I) \subset C^{1}(\bar{I})$, we have $h_{j} \in C^{1}(\bar{I})$. In particular, all $D h_{j}$ are bounded continuous functions. Thus there exists a $\beta_{0}>0$ (depending only on $h_{j}$ ) such that

$$
\sup _{x \in I}|D z(x, t)| \leq \beta_{0} \sum_{j=1}^{m}\left|z_{j}(t)\right|, \forall t \in R, P-\text { a.s. }
$$

where $z=\sum h_{j} z_{j}$ and $z_{j}$ is the Ornstein-Uhlenbeck process defined by (11). It is also easy to prove that

$$
|z|+|D z|+\left|D^{2} z\right| \leq \beta_{1} \sum_{j=1}^{m}\left|z_{j}(t)\right|,
$$

where $\beta_{1}>0$ only depends on $h_{j}$.

To show that (5) generates a random dynamical system, we let $v(t)=u_{t}(t)+\varepsilon u(t)-z(t)$, where $u, u_{t}$ is the solution of (5), then $u, v$ satisfies

$$
\left\{\begin{array}{l}
u_{t}=v-\varepsilon u+z, \\
\quad v_{t}=-\gamma A u+\varepsilon(\alpha-\varepsilon) u+(\varepsilon-\alpha) v-(i+\varepsilon \beta) u_{x} \\
\quad+\beta v_{x}+\varepsilon z+\beta z_{x}-f\left(|u|^{2}\right) u, \\
u(\tau, \omega)=u_{0}, \quad v(\tau, \omega)=u_{1}+\varepsilon u_{0}-z(\tau),
\end{array}\right.
$$

where $u_{0} \in H^{1}, \quad v_{0} \in L^{2}$, and $\phi_{0}=\phi(\tau, \omega)=\left(u_{0}, v_{0}\right)^{\mathrm{T}} \in E_{0}$.

By the same proof as deterministic case [4], one can easily get that for $\mathrm{P}-$ a.s. , $\omega \in \Omega$, the following results hold

Theorem 3.1 If $\left(u_{0}, v_{0}\right)^{\mathrm{T}} \in E_{0}$, there exists a unique solution $\phi(t, \omega)=(u(t, \omega), v(t, \omega))^{\mathrm{T}} \in E_{0}$ of (16), which satisfies

$$
u(t, \omega) \in C\left([\tau, T] ; H^{1}\right), \quad v(t, \omega) \in C\left([\tau, T] ; L^{2}\right) .
$$

If $\left(u_{0}, v_{0}\right)^{\mathrm{T}} \in E_{1}$, there exists a unique solution $\phi(t, \omega)=(u(t, \omega), v(t, \omega))^{\mathrm{T}} \in E_{1}$ of (16), which satisfies

$$
u(x, t) \in C\left([\tau, T] ; H^{2}\right), v(t, \omega) \in C\left([\tau, T] ; H^{1}\right) .
$$

From the above discussion, we denote the solution of (5) by $u(t)=u\left(t, \omega ; \tau, u_{0}\right)$ (denote sometimes by $u\left(t ; \tau, u_{0}\right), u(t ; \tau), u(t, \omega), u(t)$ or even $u$ if no confusions). Then we can define a mapping

$$
\begin{aligned}
& \varphi: R^{+} \times \Omega \times E_{0} \mapsto E_{0} \text { by } \\
& \varphi(t, \omega) \phi_{0}:=\phi\left(t, \omega ; 0, \phi_{0}\right) \\
&=\left(u\left(t, \omega ; 0, u_{0}\right), v\left(t, \omega ; 0, v_{0}\right)\right), t \geq 0,
\end{aligned}
$$

by the definition 2.2.1, it is easy to show that $\phi$ is a continuous RDS on $E_{0}$ with the following fact

$$
\varphi\left(\tau, \theta_{-\tau} \omega\right) \phi_{0}=\phi\left(0, \omega ;-\tau, \phi_{0}\right),
$$

for $\phi_{0}=\phi(\tau, \omega)=\left(u_{0}, v_{0}\right)^{\mathrm{T}} \in E_{0}, \tau \geq 0$.

\section{Random Attractors}

\subsection{Absorbing Set in $E_{0}$}

In this subsection, we prove that the RDS $\varphi$ defined by (17) has a bounded absorbing set $B(\omega) \subset E_{0}$, which absorbs, in fact, all the bounded sets $B \subset E_{0}$. Recall that $\phi\left(t, \omega ; \tau, \phi_{0}\right)=\left(u\left(t, \omega ; \tau, u_{0}\right), v\left(t, \omega ; \tau, v_{0}\right)\right)^{\mathrm{T}}$ is the solution of (16) with $u(\tau)=u_{0}$ and $v(\tau)=u_{1}+\varepsilon u_{0}-z(\tau)=v_{0}$.

We then rewrite (16) as follows 


$$
\dot{\phi}+L \phi=F(\phi, \omega), \phi(\tau, \omega)=(u(\tau, \omega), v(\tau, \omega))^{\mathrm{T}}, t \geq \tau
$$

where

$$
\begin{gathered}
\phi=\left(\begin{array}{l}
u \\
v
\end{array}\right), \quad L=\left(\begin{array}{cc}
\varepsilon I & -I \\
\gamma A-\varepsilon(\alpha-\varepsilon) I & (\alpha-\varepsilon) I
\end{array}\right), \\
F(\phi, \omega)=\left(\begin{array}{c}
z \\
-(i+\varepsilon \beta) u_{x}+\beta v_{x}+\varepsilon z+\beta z_{x}-f\left(|u|^{2}\right) u
\end{array}\right) .
\end{gathered}
$$

We now can prove the absorption of RDS $\varphi$ (defined by (17)) in $E_{0}$.

Lemma 4.1 For any no random bounded set $B$, there exists a random variable $\rho_{1}(\omega) \geq 0$ satisfying the following property: for every $\left(u_{0}, u_{1}+\varepsilon u_{0}\right)^{T} \in B$, there exists $T_{B}(\omega)<-1$, such that, for any $\tau \leq T_{B}(\omega)$, the following estimate holds $\mathrm{P}-$ a.s.

$$
\left\|\phi\left(t, \omega ; \tau, \phi_{0}\right)\right\|_{E_{0}} \leq \rho_{1}(\omega), t \in[-1,0] .
$$

Proof. Taking the inner product of (18) with $\phi$ in $E_{0}$, we obtain that

$$
\frac{1}{2} \frac{\mathrm{d}}{\mathrm{d} t}\|\phi\|^{2}+(L \phi, \phi)=(F(\phi, \omega), \phi) .
$$

Taking the real part of (19), we find that

$$
\begin{aligned}
& \frac{1}{2} \frac{\mathrm{d}}{\mathrm{d} t}\left(\|u\|^{2}+|v|^{2}\right)+\varepsilon\|u\|^{2}+(\gamma-1) \operatorname{Re}((u, v)) \\
& -\varepsilon(\alpha-\varepsilon) \operatorname{Re}(u, v)+(\alpha-\varepsilon)|v|^{2} \\
& =\operatorname{Re}((z, u))+\operatorname{Re}\left(-(i+\varepsilon \beta) u_{x}, v\right)+\beta \operatorname{Re}\left(v_{x}, v\right) \\
& \quad+\varepsilon \operatorname{Re}(z, v)+\beta \operatorname{Re}\left(z_{x}, v\right)-\operatorname{Re}\left(f\left(|u|^{2}\right) u, v\right)
\end{aligned}
$$

Since

$$
\begin{aligned}
& (\gamma-1) \operatorname{Re}((u, v)) \\
& =(\gamma-1) \operatorname{Re}\left(\left(u, u_{t}+\varepsilon u-z\right)\right) \\
& =\frac{\gamma-1}{2} \frac{\mathrm{d}}{\mathrm{d} t}\|u\|^{2}+\varepsilon(\gamma-1)\|u\|^{2}-(\gamma-1) \operatorname{Re}((u, z))
\end{aligned}
$$

and

$$
\begin{aligned}
& \operatorname{Re}\left(f\left(|u|^{2}\right) u, v\right) \\
& =\operatorname{Re}\left(f\left(|u|^{2}\right) u, u_{t}+\varepsilon u-z\right) \\
& =\operatorname{Re}\left(f\left(|u|^{2}\right) u, u_{t}\right)+\varepsilon \operatorname{Re}\left(f\left(|u|^{2}\right) u, u\right)-\operatorname{Re}\left(f\left(|u|^{2}\right) u, z\right) \\
& =\frac{1}{2} \frac{\mathrm{d}}{\mathrm{d} t} \int F\left(|u|^{2}\right) \mathrm{d} x+\varepsilon \operatorname{Re}\left(f\left(|u|^{2}\right) u, u\right)-\operatorname{Re}\left(f\left(|u|^{2}\right) u, z\right)
\end{aligned}
$$

it follows from (20)-(22), we get that

$$
\begin{aligned}
& \frac{\mathrm{d}}{\mathrm{d} t}\left(\frac{\gamma}{2}\|u\|^{2}+\frac{1}{2}|v|^{2}+\frac{1}{2} \int F\left(|u|^{2}\right) \mathrm{d} x\right)+\varepsilon \gamma\|u\|^{2} \\
& -\varepsilon(\alpha-\varepsilon) \operatorname{Re}(u, v)+(\alpha-\varepsilon)|v|^{2} \\
& +\operatorname{Re}\left((i+\varepsilon \beta) u_{x}, v\right)+\varepsilon \operatorname{Re}\left(f\left(|u|^{2}\right) u, u\right) \\
& -\gamma \operatorname{Re}((u, z))-\varepsilon \operatorname{Re}(z, v)-\beta \operatorname{Re}\left(z_{x}, v\right) \\
& -\operatorname{Re}\left(f\left(|u|^{2}\right) u, z\right)=0
\end{aligned}
$$

We introduce two functions

$$
g_{1}(u, v)=\frac{\gamma}{2}\|u\|^{2}+\frac{1}{2}|v|^{2}+\frac{1}{2} \int F\left(|u|^{2}\right) \mathrm{d} x,
$$

$$
\begin{aligned}
G_{1}(u, v)= & \varepsilon \gamma\|u\|^{2}-\varepsilon(\alpha-\varepsilon) \operatorname{Re}(u, v)+(\alpha-\varepsilon)|v|^{2} \\
& +\operatorname{Re}\left((i+\varepsilon \beta) u_{x}, v\right)+\varepsilon \operatorname{Re}\left(f\left(|u|^{2}\right) u, u\right) \\
& -\gamma \operatorname{Re}((u, z))-\varepsilon \operatorname{Re}(z, v)-\beta \operatorname{Re}\left(z_{x}, v\right), \\
& -\operatorname{Re}\left(f\left(|u|^{2}\right) u, z\right)
\end{aligned}
$$

and one process

$$
C_{1}(t)=\sum_{j=1}^{m}\left|Z_{j}(t)\right| .
$$

So that (23) gives

$$
\frac{\mathrm{d}}{\mathrm{d} t} g_{1}(u, v)+G_{1}(u, v)=0 \text {. }
$$

In the following, we denote by $c$ any constant depending only on the data $(\alpha, \beta, \gamma, \varepsilon, I, f)$, which can be different from line to line or even in the same line. Now we can prove there exist positive constants $\delta_{0}, d$ and $d_{1}$ such that

$$
\begin{gathered}
G_{1}(u, v)-\delta_{0} g_{1}(u, v) \\
=\kappa_{1}(u, v) \geq \frac{d}{2}\|\phi\|^{2}-2 \beta_{1} C_{1}(t)\|\phi\|^{2}-g(t)-c, \\
g_{1}(u, v) \geq \frac{d_{1}}{2}\|\phi\|^{2}-c,
\end{gathered}
$$

where $\beta_{1}$ is defined by (15) and $g(t)$ will be defined in the following paper, hence we obtain

$$
\frac{\mathrm{d}}{\mathrm{d} t} g_{1}(u, v)+\delta_{0} g_{1}(u, v)=-\kappa_{1}(u, v) \text {. }
$$

In fact we have

$$
\begin{aligned}
& G_{1}(u, v)-\delta_{0} g_{1}(u, v)=\kappa_{1}(u, v) \\
& =\varepsilon \gamma\|u\|^{2}-\varepsilon(\alpha-\varepsilon) \operatorname{Re}(u, v)+(\alpha-\varepsilon)|v|^{2} \\
& \quad+\operatorname{Re}\left((i+\varepsilon \beta) u_{x}, v\right)+\varepsilon \int f\left(|u|^{2}\right)|u|^{2} \mathrm{~d} x \\
& \quad-\frac{\delta_{0}}{2} \int F\left(|u|^{2}\right) \mathrm{d} x-\gamma \operatorname{Re}((u, z))-\varepsilon \operatorname{Re}(z, v) \\
& \quad-\beta \operatorname{Re}\left(z_{x}, v\right)-\operatorname{Re}\left(f\left(|u|^{2}\right) u, z\right)-\frac{\gamma \delta_{0}}{2}\|u\|^{2}-\frac{\delta_{0}}{2}|v|^{2}
\end{aligned}
$$


By estimating every terms on the right side in (31), letting

$$
\varepsilon=\frac{\alpha \gamma \lambda_{1}}{2 \alpha^{2}+3 \gamma \lambda_{1}},
$$

where $\lambda_{1}$ is the first eigenvalue of $A$, then by (32), we find that

$$
\begin{aligned}
& \varepsilon \gamma\|u\|^{2}-\varepsilon(\alpha-\varepsilon) \operatorname{Re}(u, v)+(\alpha-\varepsilon)|v|^{2} \\
& =\frac{3 \varepsilon \gamma}{4}\|u\|^{2}+\frac{\alpha}{2}|v|^{2}+\frac{\varepsilon}{2}|v|^{2}+\frac{\varepsilon \gamma}{4}\|u\|^{2}-\varepsilon(\alpha-\varepsilon) \operatorname{Re}(u, v) \\
& +\left(\frac{\alpha}{2}-\frac{3 \varepsilon}{2}\right)|v|^{2} \geq \frac{3 \varepsilon \gamma}{4}\|u\|^{2}+\frac{\alpha}{2}|v|^{2}+\frac{\varepsilon}{2}|v|^{2}+\frac{\varepsilon \gamma}{4}\|u\|^{2} \\
& -\varepsilon(\alpha-\varepsilon)|u| \cdot|v|+\left(\frac{\alpha}{2}-\frac{3 \varepsilon}{2}\right)|v|^{2} \geq \frac{3 \varepsilon \gamma}{4}\|u\|^{2}+\frac{\alpha}{2}|v|^{2}+\frac{\varepsilon}{2}|v|^{2} \\
& +\frac{\varepsilon \gamma}{4}\|u\|^{2}-\frac{\varepsilon \alpha}{\sqrt{\lambda_{1}}}\|u\| \cdot|v|+\left(\frac{\alpha}{2}-\frac{3 \varepsilon}{2}\right)|v|^{2}=\frac{3 \varepsilon \gamma}{4}\|u\|^{2}+\frac{\alpha}{2}|v|^{2} \\
& +\frac{\varepsilon}{2}|v|^{2}+\frac{\varepsilon \gamma}{4}\|u\|^{2}-\frac{\varepsilon \alpha}{\sqrt{\frac{2 \varepsilon \alpha^{2}}{\gamma(\alpha-3 \varepsilon)}}\|u\| \cdot|v|+\left(\frac{\alpha}{2}-\frac{3 \varepsilon}{2}\right)|v|^{2}} \\
& =\frac{3 \varepsilon \gamma}{4}\|u\|^{2}+\frac{\alpha}{2}|v|^{2}+\frac{\varepsilon}{2}|v|^{2}+\left(\frac{\sqrt{\varepsilon \gamma}}{2}\|u\|-\sqrt{\frac{\alpha-3 \varepsilon}{2}}|v|^{2}\right. \\
& \geq \frac{3 \varepsilon \gamma}{4}\|u\|^{2}+\frac{\alpha}{2}|v|^{2}+\frac{\varepsilon}{2}|v|^{2}
\end{aligned}
$$

and

$$
\begin{aligned}
\operatorname{Re}\left((i+\varepsilon \beta) u_{x}, v\right) & \leq \sqrt{1+\varepsilon^{2} \beta^{2}}\|u\| \cdot|v| \\
& \leq \frac{1+\varepsilon^{2} \beta^{2}}{2 \alpha}\|u\|^{2}+\frac{\alpha}{2}|v|^{2}
\end{aligned}
$$

Using $|f|_{\infty}$ uniformly bounded, we get

$$
\begin{aligned}
& -\gamma \operatorname{Re}((u, z))-\varepsilon \operatorname{Re}(z, v)-\beta \operatorname{Re}\left(z_{x}, v\right)-\operatorname{Re}\left(f\left(|u|^{2}\right) u, z\right) \\
& \leq \gamma\|z\| \cdot\|u\|+\varepsilon|z| \cdot|v|+\beta\left|z_{x}\right| \cdot|v|+\left\|f\left(|u|^{2}\right)\right\|_{L^{\infty}}|u| \cdot|z| \\
& \leq \frac{\gamma^{2} \beta_{1} C_{1}(t)}{4}+\beta_{1} C_{1}(t)\|u\|^{2}+\frac{\varepsilon^{2} \beta_{1} C_{1}(t)}{4}+\beta_{1} C_{1}(t)|v|^{2} \\
& +\frac{\beta^{2} \beta_{1} C_{1}(t)}{4}+\beta_{1} C_{1}(t)|v|^{2}+\frac{c^{2} \beta_{1} C_{1}(t)}{4 \lambda_{1}}+\beta_{1} C_{1}(t)\|u\|^{2} \\
& =2 \beta_{1} C_{1}(t)\left(\|u\|^{2}+|v|^{2}\right)+\left(\frac{\gamma^{2}}{4}+\frac{\varepsilon^{2}}{4}+\frac{\beta^{2}}{4}+\frac{c^{2}}{4 \lambda_{1}}\right) \beta_{1} C_{1}(t) \\
& =2 \beta_{1} C_{1}(t)\|\phi\|^{2}+\left(\frac{\gamma^{2}}{4}+\frac{\varepsilon^{2}}{4}+\frac{\beta^{2}}{4}+\frac{c^{2}}{4 \lambda_{1}}\right) \beta_{1} C_{1}(t) \\
& =2 \beta_{1} C_{1}(t)\|\phi\|^{2}+g(t),
\end{aligned}
$$

where $g(t)=\left(\frac{\gamma^{2}}{4}+\frac{\varepsilon^{2}}{4}+\frac{\beta^{2}}{4}+\frac{c^{2}}{4 \lambda_{1}}\right) \beta_{1} C_{1}(t)$.

Taking $\delta_{0}$, such that $\delta_{0} \leq \frac{\varepsilon}{2}$ and $\frac{2\left(1+\varepsilon^{2} \beta^{2}\right)}{\alpha \gamma} \leq \delta_{0}$, where $\varepsilon$ is defined in (32), and letting

$$
d=\min \left(\delta_{0} \gamma, \delta_{0}\right)
$$

we find that

$$
\begin{gathered}
\frac{3 \varepsilon \gamma}{4}-\frac{1+\varepsilon^{2} \beta^{2}}{2 \alpha}-\frac{\delta_{0} \gamma}{2} \geq \frac{d}{2}, \\
\frac{\alpha}{2}+\frac{\varepsilon}{2}-\frac{\alpha}{2}-\frac{\delta_{0}}{2} \geq \frac{d}{2} .
\end{gathered}
$$

Noting that $\frac{\delta_{0}}{2 \varepsilon} \leq \frac{1}{4}<1$, by (8) and (9), we have

$$
\varepsilon \int f\left(|u|^{2}\right)|u|^{2} \mathrm{~d} x-\frac{\delta_{0}}{2} \int F\left(|u|^{2}\right) \mathrm{d} x \geq \varepsilon \gamma_{0} \int|u|^{2+2 \delta} \mathrm{d} x,
$$

using

$$
\begin{aligned}
\int|u|^{2} \mathrm{~d} x & \leq(2 D)^{\frac{\delta}{1+\delta}}\left(\int|u|^{2+2 \delta} \mathrm{d} x\right)^{\frac{1}{1+\delta}} \\
& \leq \frac{\varepsilon_{0}^{1+\delta}}{1+\delta} \int|u|^{2+2 \delta} \mathrm{d} x+\frac{2 D \delta}{1+\delta} \varepsilon_{0}^{-\frac{1+\delta}{\delta}},
\end{aligned}
$$

where $\varepsilon_{0}$ is any positive number. Choosing

$$
\varepsilon_{0} \leq\left(\frac{2 \varepsilon \gamma_{0}(1+\delta)}{d}\right)^{\frac{1}{1+\delta}}
$$

we find that

$$
\varepsilon \gamma_{0} \int|u|^{2+2 \delta} \mathrm{d} x \geq \frac{d}{2}|u|^{2}-c .
$$

Combining (31)-(40), we infer that

$G_{1}(u, v)-\delta_{0} g_{1}(u, v) \geq \frac{d}{2}\|\phi\|^{2}-2 \beta_{1} C_{1}(t)\|\phi\|^{2}-g(t)-c$.

In order to prove (29), and similarly to (40), we have

$$
g_{1}(u, v) \geq \frac{\gamma}{2}\|u\|^{2}+\frac{1}{2}|v|^{2}-c .
$$

Taking $d_{1}=\min (\gamma, 1)$, we get (29).

From (27)-(30), we infer that

$$
\frac{\mathrm{d}}{\mathrm{d} t} g_{1}(u, v)+\delta_{0} g_{1}(u, v) \leq 2 \beta_{1} C_{1}(t)\|\phi\|^{2}+g(t)+c .
$$

Taking $\mu \geq \max \left(\frac{4}{\gamma}, 4\right)$, we have

$$
\frac{\mathrm{d}}{\mathrm{d} t} g_{1}(u, v) \leq\left(-\delta_{0}+\mu \beta_{1} C_{1}(t)\right) g_{1}(u, v)+g(t)+c \text {. }
$$


Putting

$$
f_{1}(t)=-\delta_{0}+\mu \beta_{1} C_{1}(t)=-\delta_{0}+\mu \beta_{1} \sum_{j=1}^{m}\left|z_{j}(t)\right|,
$$

then by the Gronwall Lemma, we get, for $t \geq \tau$

$$
g_{1}(u, v) \leq \mathrm{e}^{\int_{\tau}^{t} f_{1}(\eta) \mathrm{d} \eta} g_{1}\left(u_{0}, v_{0}\right)+\int_{\tau}^{t}(g(\eta)+c) \mathrm{e}^{f_{\eta}^{t} f_{1}(s) \mathrm{d} s} \mathrm{~d} \eta .
$$

In particular, we have, for $t \in[-1,0], \tau \leq-1$,

$$
\begin{aligned}
g_{1}(u, v) \leq & c_{1} \mathrm{e}^{\int_{\tau}^{0} f_{1}(\eta) \mathrm{d} \eta} g_{1}\left(u_{0}, v_{0}\right) \\
& +c_{1} \int_{-\infty}^{0}(g(\eta)+c) \mathrm{e}^{\int_{\eta \eta}^{0} f_{1}(s) \mathrm{d} s} \mathrm{~d} \eta,
\end{aligned}
$$

where $c_{1}=\mathrm{e}^{\delta_{0}}$, in view of the following fact: for $-1 \leq t \leq 0$,

$$
\begin{aligned}
\mathrm{e}^{\int_{\tau}^{t} f_{1}(\eta) \mathrm{d} \eta} & =\mathrm{e}^{\int_{\tau}^{0} f_{1}(\eta) \mathrm{d} \eta} \mathrm{e}^{-\int_{t}^{0} f_{1}(\eta) \mathrm{d} \eta} \\
& \leq \mathrm{e}^{\int_{\tau}^{0} f_{1}(\eta) \mathrm{d} \eta} \mathrm{e}^{\int_{t}^{0} \delta_{0} \mathrm{~d} \eta} \leq \mathrm{e}^{\delta_{0}} \mathrm{e}^{\int_{\tau}^{0} f_{1}(\eta) \mathrm{d} \eta} .
\end{aligned}
$$

To estimate all integration terms on the right side in (47), we choose $\alpha>0$ such that

$$
\mu \beta_{1} E C_{1}(0)=\mu \beta_{1} \sum_{j=1}^{m} E\left|z_{j}(0)\right|<\frac{\delta_{0}}{2} .
$$

This is possible since by (12), $E C_{1}(0) \rightarrow 0$ as $\alpha \rightarrow \infty$. Thus, since $z_{j}(t)$ is stationary and ergodic, it is easy to get

$$
\lim _{\tau \rightarrow-\infty} \frac{1}{-\tau} \int_{\tau}^{0} f_{1}(\eta) \mathrm{d} \eta=E f_{1}(0)=-\delta_{0}+\mu \beta_{1} E C_{1}(0)<-\frac{\delta_{0}}{2},
$$

which implies that

$$
\lim _{\tau \rightarrow-\infty} \mathrm{e}^{\int_{\tau}^{0} f_{1}(\eta) \mathrm{d} \eta}=0, \quad \mathrm{P} \text {-a.s. }
$$

By (13), $\frac{z_{j}(\tau)}{\tau} \rightarrow 0$ as $\tau \rightarrow-\infty$, thus $C_{1}(t)$ and further $g(t)$ is at most 1-times polynomoal growth at $-\infty$, which, together with (49), implies that

$$
q_{1}(\omega)=\int_{-\infty}^{0}(g(\eta)+c) \mathrm{e}^{\int_{\eta}^{0} f_{1}(s) \mathrm{d} s} \mathrm{~d} \eta<\infty, \quad \text { P-a.s. }
$$

and also implies that

$$
q_{2}(\omega)=\sup _{\tau \leq-1} \mathrm{e}^{\int_{\tau}^{0} f_{1}(\eta) \mathrm{d} \eta}|z(\tau)|^{2}<\infty .
$$

Noting that

$$
\begin{gathered}
g_{1}\left(u_{0}, v_{0}\right)=\left.\frac{\gamma}{2}|| u_{0}\right|^{2}+\frac{1}{2}\left|v_{0}\right|^{2}+\frac{1}{2} \int F\left(\left|u_{0}\right|^{2}\right) \mathrm{d} x, \\
\phi_{0}=\left(u_{0}, u_{1}+\varepsilon u_{0}-z(\tau)\right),
\end{gathered}
$$

and $u_{0} \in H^{1}, u_{1} \in L^{2}$, we get

$$
g_{1}\left(u_{0}, v_{0}\right) \leq c\left(\left\|\phi_{0}\right\|^{2}+1\right),
$$

then it follows from (29) and (47)-(52), we obtain that

$$
\begin{aligned}
& \frac{d_{1}}{2}\left\|\phi\left(t, \omega ; \tau, \phi_{0}\right)\right\|_{E_{0}}^{2} \\
& \leq c c_{1} \mathrm{e}^{\int_{\tau}^{0} f_{1}(\eta) \mathrm{d} \eta}\left(\left\|u_{0}\right\|^{2}+\left|u_{1}+\varepsilon u_{0}\right|^{2}+|z(\tau)|^{2}+1\right) \\
& \quad+c_{1} \int_{-\infty}^{0}(g(\eta)+c) \mathrm{e}^{\int_{\eta}^{0} f_{1}(s) \mathrm{d} s} \mathrm{~d} \eta+c .
\end{aligned}
$$

We now take

$\rho_{1}^{2}(\omega)=\frac{2 \mathrm{e}^{\delta_{0}}}{d_{1}}\left(2 c+q_{1}(\omega)+c q_{2}(\omega)\right)+\frac{2 c}{d_{1}}$ and choose $T_{B}(\omega)$ such that

$$
\mathrm{e}^{\int_{\tau}^{0} f_{1}(\eta) \mathrm{d} \eta}\left(\left\|u_{0}\right\|^{2}+\left|u_{1}+\varepsilon u_{0}\right|^{2}\right) \leq 1,
$$

for all $\tau \leq T_{B}(\omega)$, then we get

$$
\left\|\phi\left(t, \omega ; \tau, \phi_{0}\right)\right\|_{E_{0}}^{2} \leq \rho_{1}^{2}(\omega), t \in[-1,0] .
$$

\subsection{Absorbing Set in $E_{1}$}

In order to prove the absorption property in $E_{1}$, we also need the following change for (18).

Differentiating (18) with respect to $x$ and letting $\eta=u_{x}, \quad \xi=v_{x}, \quad \psi=(\eta, \xi)^{\mathrm{T}}=\left(u_{x}, v_{x}\right)^{\mathrm{T}}$, we have

$$
\begin{aligned}
& \dot{\psi}+L \psi=F(\psi, \omega), \\
& \psi(\tau, \omega)=(\eta(\tau, \omega), \xi(\tau, \omega))^{\mathrm{T}}, t \geq \tau,
\end{aligned}
$$

where

$$
L=\left(\begin{array}{cc}
\varepsilon I & -I \\
\gamma A-\varepsilon(\alpha-\varepsilon) I & (\alpha-\varepsilon) I
\end{array}\right),
$$

$$
\begin{aligned}
& F(\psi, \omega) \\
& =\left(\begin{array}{c}
z_{x} \\
-(i+\varepsilon \beta) \eta_{x}+\beta \xi_{x}+\varepsilon z_{x}+\beta z_{x x}-f^{\prime}\left(|u|^{2}\right)|u|^{2} \eta \\
-f^{\prime}\left(|u|^{2}\right) u^{2} \bar{\eta}-f\left(|u|^{2}\right) \eta
\end{array}\right) .
\end{aligned}
$$

We now can prove the absorption of RDS $\varphi$ (defined by (17)) in $E_{1}$.

Lemma 4.2 For any no random bounded set $B$, there exists a random variable $\rho_{2}(\omega) \geq 0$ satisfying the following property: for every $\left(\eta_{0}, \eta_{1}+\varepsilon \eta_{0}\right)^{T} \in B$, there exists $T_{B}(\omega)<-1$, such that, for any $\tau \leq T_{B}(\omega)$, the following estimate holds $\mathrm{P}-$ a.s.

$$
\left\|\phi\left(t, \omega ; \tau, \phi_{0}\right)\right\|_{E_{1}} \leq \rho_{2}(\omega), t \in[-1,0] .
$$

Proof. Taking the inner product of (55) with $\psi$ in $E_{0}$, we obtain that 


$$
\frac{1}{2} \frac{\mathrm{d}}{\mathrm{d} t}\|\psi\|^{2}+(L \psi, \psi)=(F(\psi, \omega), \psi) .
$$

Taking the real part of (56), we find that

$\frac{1}{2} \frac{\mathrm{d}}{\mathrm{d} t}\left(\|\eta\|^{2}+|\xi|^{2}\right)+\varepsilon\|\eta\|^{2}+(\gamma-1) \operatorname{Re}((\eta, \xi))$

$-\varepsilon(\alpha-\varepsilon) \operatorname{Re}(\eta, \xi)+(\alpha-\varepsilon)|\xi|^{2}$

$=\operatorname{Re}\left(\left(z_{x}, \eta\right)\right)+\operatorname{Re}\left(-(i+\varepsilon \beta) \eta_{x}, \xi\right)+\beta \operatorname{Re}\left(\xi_{x}, \xi\right)$

$+\varepsilon \operatorname{Re}\left(z_{x}, \xi\right)+\beta \operatorname{Re}\left(z_{x x}, \xi\right)-\operatorname{Re}\left(f^{\prime}\left(|u|^{2}\right)|u|^{2} \eta, \xi\right)$

$-\operatorname{Re}\left(f^{\prime}\left(|u|^{2}\right) u^{2} \bar{\eta}, \xi\right)-\operatorname{Re}\left(f\left(|u|^{2}\right) \eta, \xi\right)$.

Due to

$$
\begin{aligned}
& (\gamma-1) \operatorname{Re}((\eta, \xi))=(\gamma-1) \operatorname{Re}\left(\left(\eta, \eta_{t}+\varepsilon \eta-z_{x}\right)\right) \\
& =\frac{\gamma-1}{2} \frac{\mathrm{d}}{\mathrm{d} t}\|\eta\|^{2}+\varepsilon(\gamma-1)\|\eta\|^{2}-(\gamma-1) \operatorname{Re}\left(\left(\eta, z_{x}\right)\right),
\end{aligned}
$$

and

$$
\begin{aligned}
& \operatorname{Re}\left(f^{\prime}\left(|u|^{2}\right)|u|^{2} \eta, \xi\right)=\operatorname{Re}\left(f^{\prime}\left(|u|^{2}\right)|u|^{2} \eta, \eta_{t}+\varepsilon \eta-z_{x}\right) \\
& =\frac{1}{2} \frac{\mathrm{d}}{\mathrm{d} t} \int f^{\prime}\left(|u|^{2}\right)|u|^{2}|\eta|^{2} \mathrm{~d} x-\int f^{\prime}\left(|u|^{2}\right)|\eta|^{2} \operatorname{Re}\left(u \bar{u}_{t}\right) \mathrm{d} x \\
& \quad-\int f^{\prime \prime}\left(|u|^{2}\right)|u|^{2}|\eta|^{2} \operatorname{Re}\left(u \bar{u}_{t}\right) \mathrm{d} x+\varepsilon \int f^{\prime}\left(|u|^{2}\right)|u|^{2}|\eta|^{2} \mathrm{~d} x \\
& \quad-\operatorname{Re}\left(f^{\prime}\left(|u|^{2}\right)|u|^{2} \eta, z_{x}\right),
\end{aligned}
$$

and

$$
\begin{aligned}
& \operatorname{Re}\left(f\left(|u|^{2}\right) \eta, \xi\right)=\operatorname{Re}\left(f\left(|u|^{2}\right) \eta, \eta_{t}+\varepsilon \eta-z_{x}\right) \\
& =\frac{1}{2} \frac{\mathrm{d}}{\mathrm{d} t} \int f\left(|u|^{2}\right)|\eta|^{2} \mathrm{~d} x-\int f^{\prime}\left(|u|^{2}\right)|\eta|^{2} \operatorname{Re}\left(u \bar{u}_{t}\right) \mathrm{d} x \\
& \quad+\varepsilon \int f\left(|u|^{2}\right)|\eta|^{2} \mathrm{~d} x-\operatorname{Re}\left(f\left(|u|^{2}\right) \eta, z_{x}\right) .
\end{aligned}
$$

In view of (57)-(60), we get that

$\frac{\mathrm{d}}{\mathrm{d} t}\left(\frac{\gamma}{2}\|\eta\|^{2}+\frac{1}{2}|\xi|^{2}+\frac{1}{2} \int f^{\prime}\left(|u|^{2}\right)|u|^{2}|\eta|^{2} d x\right.$

$\left.+\frac{1}{2} \int f\left(|u|^{2}\right)|\eta|^{2} d x\right)+\varepsilon \gamma\|\eta\|^{2}-\varepsilon(\alpha-\varepsilon) \operatorname{Re}(\eta, \xi)$

$+(\alpha-\varepsilon)|\xi|^{2}-\gamma \operatorname{Re}\left(\left(\eta, z_{x}\right)\right)+\operatorname{Re}\left((i+\varepsilon \beta) \eta_{x}, \xi\right)$

$-\varepsilon \operatorname{Re}\left(z_{x}, \xi\right)-\beta \operatorname{Re}\left(z_{x x}, \xi\right)+\operatorname{Re}\left(f^{\prime}\left(|u|^{2}\right) u^{2} \bar{\eta}, \xi\right)$

$-2 \int f^{\prime}\left(|u|^{2}\right)|\eta|^{2} \operatorname{Re}\left(u \bar{u}_{t}\right) \mathrm{d} x-\int f^{\prime \prime}\left(|u|^{2}\right)|u|^{2}|\eta|^{2} \operatorname{Re}\left(u \bar{u}_{t}\right) \mathrm{d} x$

$+\varepsilon \int f^{\prime}\left(|u|^{2}\right)|u|^{2}|\eta|^{2} \mathrm{~d} x-\operatorname{Re}\left(f^{\prime}\left(|u|^{2}\right)|u|^{2} \eta, z_{x}\right)$

$+\varepsilon \int f\left(|u|^{2}\right)|\eta|^{2} \mathrm{~d} x-\operatorname{Re}\left(f\left(|u|^{2}\right) \eta, z_{x}\right)=0$.

\section{Letting}

$$
\begin{aligned}
g_{2}(\eta, \xi)= & \frac{\gamma}{2}\|\eta\|^{2}+\frac{1}{2}|\xi|^{2}+\frac{1}{2} \int f^{\prime}\left(|u|^{2}\right)|u|^{2}|\eta|^{2} \mathrm{~d} x \\
& +\frac{1}{2} \int f\left(|u|^{2}\right)|\eta|^{2} \mathrm{~d} x
\end{aligned}
$$

and

$$
\begin{aligned}
& G_{2}(\eta, \xi) \\
& =\varepsilon \gamma\|\eta\|^{2}-\varepsilon(\alpha-\varepsilon) \operatorname{Re}(\eta, \xi)+(\alpha-\varepsilon)|\xi|^{2}-\gamma \operatorname{Re}\left(\left(\eta, z_{x}\right)\right) \\
& +\operatorname{Re}\left((i+\varepsilon \beta) \eta_{x}, \xi\right)-\varepsilon \operatorname{Re}\left(z_{x}, \xi\right)-\beta \operatorname{Re}\left(z_{x x}, \xi\right) \\
& +\operatorname{Re}\left(f^{\prime}\left(|u|^{2}\right) u{ }^{2} \bar{\eta}, \xi\right)-2 \int f^{\prime}\left(|u|^{2}\right)|\eta|^{2} \operatorname{Re}\left(u \bar{u}_{t}\right) \mathrm{d} x \\
& -\int f^{\prime \prime}\left(|u|^{2}\right)|u|^{2}|\eta|^{2} \operatorname{Re}\left(u \bar{u}_{t}\right) \mathrm{d} x+\varepsilon \int f^{\prime}\left(|u|^{2}\right)|u|^{2}|\eta|^{2} \mathrm{~d} x \\
& -\operatorname{Re}\left(f^{\prime}\left(|u|^{2}\right)|u|^{2} \eta, z_{x}\right)+\varepsilon \int f\left(|u|^{2}\right)|\eta|^{2} \mathrm{~d} x \\
& -\operatorname{Re}\left(f\left(|u|^{2}\right) \eta, z_{x}\right)
\end{aligned}
$$

Then it comes from (61)-(63) that

$$
\frac{\mathrm{d}}{\mathrm{d} t} g_{2}(\eta, \xi)+G_{2}(\eta, \xi)=0 .
$$

Now similarly to the above arguments (Lemma 4.1), we can prove that there exist $\delta_{2}>0, d_{2}>0$ and $d_{3}>0$, such that

$$
\begin{aligned}
G_{2}(\eta, \xi)-\delta_{2} g_{2}(\eta, \xi) & \\
=\kappa_{2}(\eta, \xi) \geq & \frac{d_{2}}{2}\|\psi\|^{2}-2 \beta_{1} C_{1}(t)\|\psi\|^{2}-g_{0}(t)-c, \\
& g_{2}(\eta, \xi) \geq \frac{d_{3}}{2}\|\psi\|^{2}-c,
\end{aligned}
$$

where $\beta_{1}$ is defined by (15) and $g_{0}(t)$ will be defined in the following paper, therefore we have

$$
\frac{\mathrm{d}}{\mathrm{d} t} g_{2}(\eta, \xi)+\delta_{2} g_{2}(\eta, \xi)=-\kappa_{2}(\eta, \xi) .
$$

In fact,

$$
\begin{aligned}
& G_{2}(\eta, \xi)-\delta_{2} g_{2}(\eta, \xi)=\kappa_{2}(\eta, \xi) \\
& =\varepsilon \gamma \|\left.\eta\right|^{2}-\varepsilon(\alpha-\varepsilon) \operatorname{Re}(\eta, \xi)+(\alpha-\varepsilon)|\xi|^{2} \\
& +\operatorname{Re}\left((i+\varepsilon \beta) \eta_{x}, \xi\right)-\gamma \operatorname{Re}\left(\left(\eta, z_{x}\right)\right)-\varepsilon \operatorname{Re}\left(z_{x}, \xi\right) \\
& -\beta \operatorname{Re}\left(z_{x x}, \xi\right)+\operatorname{Re}\left(f^{\prime}\left(|u|^{2}\right) u^{2} \bar{\eta}, \xi\right) \\
& -2 \int f^{\prime}\left(|u|^{2}\right)|\eta|^{2} \operatorname{Re}\left(u \bar{u}_{t}\right) \mathrm{d} x-\int f^{\prime \prime}\left(|u|^{2}\right)|u|^{2}|\eta|^{2} \operatorname{Re}\left(u \bar{u}_{t}\right) \mathrm{d} x \\
& +\varepsilon \int f^{\prime}\left(|u|^{2}\right)|u|^{2}|\eta|^{2} \mathrm{~d} x-\operatorname{Re}\left(f^{\prime}\left(|u|^{2}\right)|u|^{2} \eta, z_{x}\right) \\
& +\varepsilon \int f\left(|u|^{2}\right)|\eta|^{2} \mathrm{~d} x-\operatorname{Re}\left(f\left(|u|^{2}\right) \eta, z_{x}\right)-\left.\frac{\gamma \delta_{2}}{2}\left|\eta \|^{2}-\frac{\delta_{2}}{2}\right| \xi\right|^{2} \\
& -\frac{\delta_{2}}{2} \int f^{\prime}\left(|u|^{2}\right)|u|^{2}|\eta|^{2} \mathrm{~d} x-\frac{\delta_{2}}{2} \int f\left(|u|^{2}\right)|\eta|^{2} \mathrm{~d} x .
\end{aligned}
$$


Taking $\delta_{2}, d_{2}$ are the same as $\delta_{0}, d$ respectively in (36) and using $|\eta|,\left|u_{t}\right|,|u|_{\infty},|f|_{\infty},\left|f^{\prime}\right|_{\infty}$, $\left\|||^{2} f^{\prime \prime}\left(|u|^{2}\right)\right\|_{\infty}$ and uniformly bounded in time, we majoring every term on the right side in (68) to get

$$
\begin{aligned}
& \varepsilon \gamma\|\eta\|^{2}-\varepsilon(\alpha-\varepsilon) \operatorname{Re}(\eta, \xi)+(\alpha-\varepsilon)|\xi|^{2} \\
= & \frac{3 \varepsilon \gamma}{4}\|\eta\|^{2}+\frac{\alpha}{2}|\xi|^{2}+\frac{\varepsilon}{2}|\xi|^{2}+\frac{\varepsilon \gamma}{4}\|\eta\|^{2} \\
& -\varepsilon(\alpha-\varepsilon) \operatorname{Re}(\eta, \xi)+\left(\frac{\alpha}{2}-\frac{3 \varepsilon}{2}\right)|\xi|^{2} \\
\geq & \frac{3 \varepsilon \gamma}{4}\|\eta\|^{2}+\frac{\alpha}{2}|\xi|^{2}+\frac{\varepsilon}{2}|\xi|^{2}+\frac{\varepsilon \gamma}{4}\|\eta\|^{2} \\
& -\varepsilon(\alpha-\varepsilon)|\eta| \cdot|\xi|+\left(\frac{\alpha}{2}-\frac{3 \varepsilon}{2}\right)|\xi|^{2} \\
\geq & \frac{3 \varepsilon \gamma}{4}\|\eta\|^{2}+\frac{\alpha}{2}|\xi|^{2}+\frac{\varepsilon}{2}|\xi|^{2}+\frac{\varepsilon \gamma}{4}\|\eta\|^{2} \\
& -\frac{\varepsilon \alpha}{\sqrt{\lambda}}\|\eta\| \cdot|\xi|+\left(\frac{\alpha}{2}-\frac{3 \varepsilon}{2}\right)|\xi|^{2} \\
\geq & \frac{3 \varepsilon \gamma}{4}\|\eta\|^{2}+\frac{\alpha}{2}|\xi|^{2}+\frac{\varepsilon}{2}|\xi|^{2},
\end{aligned}
$$

where $\varepsilon$ is defined by (32) and $\lambda_{1}$ is the first eigenvalue of $A$, and we have

$$
\begin{aligned}
\operatorname{Re}\left((i+\varepsilon \beta) \eta_{x}, \xi\right) & \leq \sqrt{1+\varepsilon^{2} \beta^{2}}\|\eta\| \cdot|\xi| \\
& \leq \frac{1+\varepsilon^{2} \beta^{2}}{\alpha}\|\eta\|^{2}+\frac{\alpha}{4}|\xi|^{2}
\end{aligned}
$$

and

$$
\begin{aligned}
& -\gamma \operatorname{Re}\left(\left(\eta, z_{x}\right)\right)-\varepsilon \operatorname{Re}\left(z_{x}, \xi\right)-\beta \operatorname{Re}\left(z_{x x}, \xi\right) \\
& -\operatorname{Re}\left(f\left(|u|^{2}\right) \eta, z_{x}\right)-\operatorname{Re}\left(f^{\prime}\left(|u|^{2}\right)|u|^{2} \eta, z_{x}\right) \\
& \leq \gamma\|\eta\| \cdot\left\|z_{x}\right\|+\varepsilon\left|z_{x}\right| \cdot|\xi|+\beta\left|z_{x x}\right| \cdot|\xi| \\
& +\left\|\left.f\left(|u|^{2}\right)\right|_{L^{\infty}}|\eta| \cdot\left|z_{x}\right|+\right\| f^{\prime}\left(|u|^{2}\right)|u|^{2} \|_{L^{\infty}}|\eta| \cdot\left|z_{x}\right| \\
& \leq \frac{\gamma^{2} \beta_{1} C_{1}(t)}{8}+2 \beta_{1} C_{1}(t)\|\eta\|^{2}+\frac{\varepsilon^{2} \beta_{1} C_{1}(t)}{4}+\beta_{1} C_{1}(t)|\xi|^{2} \\
& +\frac{\beta^{2} \beta_{1} C_{1}(t)}{4}+\beta_{1} C_{1}(t)|\xi|^{2}+c \beta_{1} C_{1}(t) \\
& =2 \beta_{1} C_{1}(t)\|\psi\|^{2}+\left(\frac{\gamma^{2}}{8}+\frac{\varepsilon^{2}}{4}+\frac{\beta^{2}}{4}+c\right) \beta_{1} C_{1}(t) \\
& =2 \beta_{1} C_{1}(t)\|\psi\|^{2}+g_{0}(t),
\end{aligned}
$$

where $g_{0}(t)=\left(\frac{\gamma^{2}}{8}+\frac{\varepsilon^{2}}{4}+\frac{\beta^{2}}{4}+c\right) \beta_{1} C_{1}(t)$.

By $|\eta|_{L^{4}}^{2} \leq c\left|\eta_{x}\right|^{1 / 2}|\eta|^{3 / 2}$ and young inequality, we have

$$
\begin{gathered}
\operatorname{Re}\left(f^{\prime}\left(|u|^{2}\right) u^{2} \bar{\eta}, \xi\right) \\
\leq\left\|f^{\prime}\left(|u|^{2}\right)|u|^{2}\right\|_{L_{\infty}}|\eta| \cdot|\xi| \leq c|\eta|^{2}+\frac{\alpha}{4}|\xi|^{2}, \\
-2 \int f^{\prime}\left(|u|^{2}\right)|\eta|^{2} \operatorname{Re}\left(u \bar{u}_{t}\right) \mathrm{d} x \\
\leq 2\left\|f^{\prime}\left(|u|^{2}\right)\right\|_{L^{\infty}}|u|_{L^{\infty}}|\eta|_{L^{4}}^{2}\left|u_{t}\right| \leq \frac{d_{2}}{8}\|\eta\|^{2}+c|\eta|^{2} \\
-\int f^{\prime \prime}\left(|u|^{2}\right)|u|^{2}|\eta|^{2} \operatorname{Re}\left(u \bar{u}_{t}\right) \mathrm{d} x \\
\leq\left\|f^{\prime \prime}\left(|u|^{2}\right)|u|^{2}\right\|_{L^{\infty}}|u|_{L^{\infty}}|\eta|_{L^{4}}^{2}\left|u_{t}\right| \leq \frac{d_{2}}{8}\|\eta\|^{2}+c|\eta|^{2}, \\
\left(\varepsilon-\frac{\delta_{2}}{2}\right) \int f^{\prime}\left(|u|^{2}\right)|u|^{2}|\eta|^{2} \mathrm{~d} x \\
\leq\left(\varepsilon-\frac{\delta_{2}}{2}\right)\left\|f^{\prime}\left(|u|^{2}\right)|u|^{2}\right\|_{L^{\infty}}|\eta|_{L^{4}}^{2} \leq \frac{d_{2}}{8}\|\eta\|^{2}+c|\eta|^{2} \\
\left(\varepsilon-\frac{\delta_{2}}{2}\right) \int f\left(|u|^{2}\right)|\eta|^{2} \mathrm{~d} x \\
\leq\left(\varepsilon-\frac{\delta_{2}}{2}\right)\left\|f\left(|u|^{2}\right)\right\|\left\|_{L^{\infty}}|\eta|_{L^{4}}^{2} \leq \frac{d_{2}}{8}\right\| \eta \|^{2}+c|\eta|^{2}
\end{gathered}
$$

Then it follows from (68)-(76) that

$$
\begin{aligned}
& G_{2}(\eta, \xi)-\delta_{2} g_{2}(\eta, \xi) \\
& \geq \frac{d_{2}}{2}\|\psi\|^{2}-2 \beta_{1} C_{1}(t)\|\psi\|^{2}-g_{0}(t)-c|\eta|^{2} . \\
& \geq \frac{d_{2}}{2}\|\psi\|^{2}-2 \beta_{1} C_{1}(t)\|\psi\|^{2}-g_{0}(t)-c
\end{aligned}
$$

Similarly, with $d_{3}=\min (\gamma, 1)$, we can easily derive that

$$
g_{2}(\eta, \xi) \geq \frac{d_{3}}{2}\|\psi\|^{2}-c .
$$

From (64)-(67), we infer that

$$
\frac{\mathrm{d}}{\mathrm{d} t} g_{2}(\eta, \xi)+\delta_{2} g_{2}(\eta, \xi) \leq 2 \beta_{1} C_{1}(t)\|\psi\|^{2}+g_{0}(t)+c,
$$

it follows from (44) that, for the same $\mu$, we have

$$
\frac{\mathrm{d}}{\mathrm{d} t} g_{2}(\eta, \xi) \leq\left(-\delta_{2}+\mu \beta_{1} C_{1}(t)\right) g_{2}(\eta, \xi)+g_{0}(t)+c \text {. }
$$

Putting

$$
f_{2}(t)=-\delta_{2}+\mu \beta_{1} C_{1}(t)=-\delta_{2}+\mu \beta_{1} \sum_{j=1}^{m}\left|z_{j}(t)\right|,
$$

then by the Gronwall Lemma, we have, for $t \geq \tau$

$$
\begin{aligned}
g_{2}(\eta, \xi) \leq & \mathrm{e}^{\int_{\tau}^{t} f_{2}(\sigma) \mathrm{d} \sigma} g_{2}\left(\eta_{0}, \xi_{0}\right) \\
& +\int_{\tau}^{t}\left(g_{0}(\sigma)+c\right) \mathrm{e}^{\int_{\sigma}^{t} f_{2}(s) \mathrm{d} s} \mathrm{~d} \sigma .
\end{aligned}
$$


The same as (47), we have, for $t \in[-1,0], \tau \leq-1$,

$$
\begin{aligned}
g_{2}(\eta, \xi) \leq & c_{1} \mathrm{e}^{\int_{\tau}^{0} f_{2}(\sigma) \mathrm{d} \sigma} g_{2}\left(\eta_{0}, \xi_{0}\right) \\
& +c_{1} \int_{-\infty}^{0}\left(g_{0}(\sigma)+c\right) \mathrm{e}^{\int_{\sigma}^{0} f_{2}(s) \mathrm{ds}} \mathrm{d} \sigma
\end{aligned}
$$

where $c_{1}=\mathrm{e}^{\delta_{2}}$, and

$$
\begin{aligned}
& q_{1}^{\prime}(\omega)=\int_{-\infty}^{0}\left(g_{0}(\sigma)+c\right) \mathrm{e}^{\int_{\sigma}^{0} f_{2}(s) \mathrm{d} s} \mathrm{~d} \sigma<\infty, \\
& q_{2}^{\prime}(\omega)=\sup _{\tau \leq-1} \mathrm{e}_{\tau}^{\int_{\tau} f_{2}(\sigma) \mathrm{d} \sigma}\left|z_{x}(\tau)\right|^{2}<\infty .
\end{aligned}
$$

Noting that

$$
\begin{aligned}
& g_{2}\left(\eta_{0}, \xi_{0}\right) \\
& =\frac{\gamma}{2}\left\|\eta_{0}\right\|^{2}+\frac{1}{2}\left|\xi_{0}\right|^{2}+\frac{1}{2} \int f^{\prime}\left(\left|u_{0}\right|^{2}\right)\left|u_{0}\right|^{2}\left|\eta_{0}\right|^{2} \mathrm{~d} x, \\
& +\frac{1}{2} \int f\left(\left|u_{0}\right|^{2}\right)\left|\eta_{0}\right|^{2} \mathrm{~d} x \\
& \psi_{0}=\left(\eta_{0}, \eta_{1}+\varepsilon \eta_{0}-z_{x}(\tau)\right),
\end{aligned}
$$

we get

$$
g_{2}\left(\eta_{0}, \xi_{0}\right) \leq c\left(\left\|\psi_{0}\right\|^{2}+1\right),
$$

then from (66) and (83)-(86), we obtain that

$$
\begin{aligned}
& \frac{d_{2}}{2}\left\|\psi\left(t, \omega ; \tau, \psi_{0}\right)\right\|_{E_{0}}^{2} \\
& \leq c c_{1} \mathrm{e}^{\int_{\tau}^{0} f_{2}(\sigma) \mathrm{d} \sigma}\left(\left\|\eta_{0}\right\|^{2}+\left|\eta_{1}+\varepsilon \eta_{0}\right|^{2}+\left|z_{x}(\tau)\right|^{2}+1\right) . \\
& \quad+c_{1} \int_{-\infty}^{0}\left(g_{0}(\sigma)+c\right) \mathrm{e}^{\int_{\sigma}^{0} f_{2}(s) \mathrm{d} s} \mathrm{~d} \sigma+c
\end{aligned}
$$

We now take

$\rho_{2}^{2}(\omega)=\frac{2 \mathrm{e}^{\delta_{2}}}{d_{2}}\left(2 c+q_{1}^{\prime}(\omega)+c q_{2}^{\prime}(\omega)\right)+\frac{2 c}{d_{2}}$ and choose $T_{B}(\omega)$ such that

$$
\mathrm{e}^{\int_{\tau}^{0} f_{2}(\sigma) \mathrm{d} \sigma}\left(\left\|\eta_{0}\right\|^{2}+\left|\eta_{1}+\varepsilon \eta_{0}\right|^{2}\right) \leq 1,
$$

for all $\tau \leq T_{B}(\omega)$, then we get

$$
\left\|\phi\left(t, \omega ; \tau, \phi_{0}\right)\right\|_{E_{1}}^{2} \leq \rho_{2}^{2}(\omega), \quad t \in[-1,0] .
$$

\subsection{The Compactness in $E_{0}$}

In this subsection, we prove the compactness in $E_{0}$ through the decomposition of solution semigroup.

Let $u(t)$ be a solution of (5) with initial value $\left(u_{0}, u_{1}+\varepsilon u_{0}-z(\tau)\right)^{\mathrm{T}}$. We make the decomposition $u(t)=y_{1}(t)+y_{2}(t)$, where $y_{1}(t)$ and $y_{2}(t)$ satisfy

$$
\left\{\begin{array}{l}
d y_{1 t}+\alpha y_{1 t} d t-\beta y_{1 x t} d t-\gamma y_{1 x x} d t+i y_{1 x} d t=0, \\
y_{1}(x, \tau)=u_{0}(x), \quad y_{1 t}(x, \tau)=u_{1}(x), \\
y_{1}(x-L, t)=y_{1}(x+L, t) .
\end{array}\right.
$$

and

$$
\left\{\begin{aligned}
& d y_{2 t}+\alpha y_{2 t} d t- \beta y_{2 x t} d t-\gamma y_{2 x x} d t+i y_{2 x} d t \\
&+f\left(|u|^{2}\right) u d t=\sum_{j=1}^{m} h_{j} d W_{j}, \\
& y_{2}(x, \tau)=0, \quad y_{2 t}(x, \tau)=0 \\
& y_{2}(x-L, t)=y_{2}(x+L, t) .
\end{aligned}\right.
$$

Lemma 4.3 For any no random bounded set $B$, we have, for any $\left(u_{0}, u_{1}+\varepsilon u_{0}\right)^{\mathrm{T}} \in B$

$$
\begin{aligned}
\left\|Y_{1}(0)\right\|_{E_{0}}^{2} & =\left\|y_{1}(0)\right\|^{2}+\left|y_{1 t}(0)+\varepsilon y_{1}(0)\right|^{2} \\
& \leq \frac{2}{d_{1}}\left(\left\|u_{0}\right\|^{2}+\left|u_{1}+\varepsilon u_{0}\right|^{2}\right) \mathrm{e}^{\delta_{3} \tau},
\end{aligned}
$$

and there exists a random variable $\rho_{3}(\omega) \geq 0$ such that for $\mathrm{P}-$ a.s. $\omega \in \Omega$

$$
\left\|D Y_{2}\left(0, \omega ; \tau, Y_{2}(\tau, \omega)\right)\right\|_{E_{0}}^{2} \leq \rho_{3}^{2}(\omega),
$$

where $Y_{1}=\left(y_{1}, y_{1 t}+\varepsilon y_{1}\right)^{\mathrm{T}}$ and $Y_{2}=\left(y_{2}, y_{2 t}+\varepsilon y_{2}-z\right)^{\mathrm{T}^{1}}$ satisfy (89) and (90) respecttively.

Proof. Taking the inner product of (89) with $Y_{1}$ in $E_{0}$ whose initial value is $\left(u_{0}, u_{1}+\varepsilon u_{0}\right)^{\mathrm{T}}$, after a simple computation similarly as Lemma 4.1, we obtain (91).

Taking the inner product of (90) with $\mathrm{AY}_{2}$ in $E_{0}$ whose initial value is $(0,-z(\tau))$, after a simple computation similarly as Lemma 4.2, we obtain (92).

Let $B_{1 / 2}(\omega)$ be the ball of $E_{1}=H^{2} \times H^{1}$ of random variable $\rho_{3}(\omega) \geq 0$. From the compact embedding $E_{1}=H^{2} \times H^{1} \rightarrow E_{0}=H^{1} \times L^{2}$, we see that $B_{1 / 2}(\omega)$ is compact. For any no random bounded set $B$ of $E_{0}$, pick any $\phi(0) \in \varphi\left(t, \theta_{-t} \omega\right) B$. From Lemma 4.3 , we have $Y_{2}(0)=\phi(0)-Y_{1}(0) \in B_{1 / 2}(\omega)$, where $Y_{2}(t, \omega)$ is given by Lemma 4.3. Therefore, again by Lemma 4.3,

$$
\begin{aligned}
& \inf _{\ell(0) \in B_{1 / 2}(\omega)}\|\phi(0)-\ell(0)\|_{E_{0}}^{2} \\
& \leq\left\|Y_{1}(0)\right\|_{E_{0}}^{2} \leq \frac{2}{d_{1}}\left(\left\|u_{0}\right\|^{2}+\left|u_{1}+\varepsilon u_{0}\right|^{2}\right) \mathrm{e}^{\delta_{3} \tau}, \tau \leq 0 .
\end{aligned}
$$

So

$$
\operatorname{dist}\left(\varphi\left(t, \theta_{-t} \omega\right) B, B_{1 / 2}(\omega)\right) \rightarrow 0, \text { as } t \rightarrow+\infty .
$$

Corollary 4.4 The RDS $\varphi(t, \omega)$ associated with (17) possesses a uniformly attracting compact set $B_{1 / 2}(\omega) \subset E_{0}$, so the RDS $\varphi(t, \omega)$ is uniformly asymptotically compact in $E_{0}$.

By applying Theorem 2.2.2, Lemma 4.1 and corollary 4.4, we obtain the final conclusion of this whole paper.

Theorem 4.5 Assume $h_{j} \in D(A)=H^{1} \cap H^{2}$, then the RDS $\varphi$ modeling the dissipative Hamiltonian amplitude equation governing modulated wave instabilities possesses a compact random attractor $\mathrm{A}(\omega)$ which 
attracts all bounded sets of $E_{0}=H^{1} \times L^{2}$.

\section{Acknowledgements}

The authors would like to express their sincere thanks to the anonymous referee for his/her valuable comments and suggestions to improve the paper.

\section{REFERENCES}

[1] M. Tanaka and N. Yajima, "Soliton Modes in an Unstable Plasma Nonlinear Phenomena in an Electron-Beam Plasma," Progress of Theoretical Physics Supplement, Vol. 94, 1988, pp. 138-162.

[2] T. Yajima and M. Wadati, "Solitons in an Unstable Medium,” Journal of the Physical Society of Japan, Vol. 56, 1987, pp. 3069-3081.

[3] M. Wadati, H. Segur and M. J. Ablowitz, “A New Hamiltonian Amplitude Equation Governing Modulated Wave Instabilities," Journal of the Physical Society of Japan, Vol. 61, No. 4, 1992, pp. 1187-1193. doi:10.1143/JPSJ.61.1187

[4] B. L. Guo and Z. D. Dai, “Attractor for the Dissipative Hamiltonian Amplitude Equation Governing Modulated Wave Instabilities," Discrete and Continuous Dynamical Systems, Vol. 4, No. 4, 1998, pp. 783-793. doi:10.3934/dcds.1998.4.783

[5] Z. D. Dai, "Regularity of the Attractor for the Dissipative Hamiltonian Amplitude Equation Governing Modulated Wave Instabilities," Acta Mathematicae Applicatae Sinica (English Series), Vol. 18, No. 2, 2002, pp. 263-272. doi:10.1007/s102550200025

[6] Z. D. Dai, L. Yang and J. Huang, "Attractor for the Un- perturbed Dissipative Hamiltonian Amplitude Wave," Acta Mathematicae Applicatae Sinica, Vol. 27, No. 4, 2004, pp. 577-592.

[7] L. Yang and Z. D. Dai, "Finite Dimension of Global Attractors for Dissipative Equations Governing Modulated Wave," Applied Mathematics: A Journal of Chinese Universities, Vol. 18, No. 4, 2003, pp. 421-430. doi:10.1007/s11766-003-0069-3

[8] Y. R. Li and B. L. Guo, "Random Attractors of Boussinesq Equations with Multiplicative Noise,” Acta Mathematicae Sinica (English Series), Vol. 25, No. 3, 2009, pp. 481-490. doi:10.1007/s10114-008-6226-0

[9] Y. R. Li, and B. L. Guo, "Random Attractors for QuasiContinuous Random Dynamical Systems and Applications to Stochastic Reaction-Diffusion Equations,” Journal of Differential Equations, Vol. 245, No. 7, 2008, pp. 1775-1800. doi:10.1016/j.jde.2008.06.031

[10] X. M. Fan, "Random Attractor for a Damped SineGordon Equation with White Noise," Pacific Journal of Mathematics, Vol. 216, No. 1, 2004, pp. 63-76. doi:10.2140/pjm.2004.216.63

[11] H. Crauel and F. Flandoli, "Attracors for Random Dynamical Systems,” Probability Theory and Related Fields, Vol. 100, No. 3, 1994, pp. 365-393. doi:10.1007/BF01193705

[12] H. Crauel and F. Flandoli, "Random Attractors," Journal of Dynamics and Differential Equations, Vol. 9, No. 2, 1997, pp. 307-341. doi:10.1007/BF02219225

[13] L. Arnold, "Random Dynamical Systems," SpringerVerlag, New York, 1998.

[14] R. Temam, "Infinite-Dimensional Dynamical System in Mechanics and Physics,” Springer-Verlag, New York, 1988, pp. 90-226. doi:10.1007/978-1-4684-0313-8 